\title{
How Long Do Endoprosthetic Reconstructions for Proximal Femoral Tumors Last?
}

\author{
Nicholas M. Bernthal MD, Adam J. Schwartz MD, \\ Daniel A. Oakes MD, J. Michael Kabo PhD, \\ Jeffrey J. Eckardt MD
}

Published online: 4 May 2010

(c) The Author(s) 2010. This article is published with open access at Springerlink.com

\begin{abstract}
Background As the life expectancy of patients with musculoskeletal tumors improves, long-term studies of endoprosthetic reconstructions are necessary to establish realistic expectations for the implants and compare them to other reconstruction approaches.

Questions/purposes (1) What is the long-term survival of cemented bipolar proximal femoral replacements? (2) How does prosthesis survival compare to patient survival among patients with Stage I, II, and III disease? (3) Do modular implants outperform custom-built prostheses? (4) Do some proximal femoral replacements require conversion to THA?

Patients and Methods We retrospectively reviewed all 86 proximal femoral replacements used for tumor reconstruction from 1982 to 2008. Primary diagnoses were 43 high-grade tumors (IIA/IIB), 20 low-grade tumors (IA/IB
\end{abstract}

Each author certifies that he or she has no commercial associations (eg, consultancies, stock ownership, equity interest, patent/licensing arrangements, etc) that might pose a conflict of interest in connection with the submitted article.

Each author certifies that his or her institution approved the human protocol for this investigation, that all investigations were conducted in conformity with ethical principles of research, and that informed consent for participation in the study was obtained.

This work was performed at University of California Los Angeles Medical Center.

N. M. Bernthal ( $₫)$, A. J. Schwartz, D. A. Oakes, J. J. Eckardt Department of Orthopaedic Surgery, University of California Los Angeles Medical Center, 1250 16th Street, Room 745,

Santa Monica, CA 90404, USA

e-mail: nbernthal@mednet.ucla.edu

J. M. Kabo

College of Engineering and Computer Science, California State

University, Northridge, CA, USA or benign), and 23 with metastatic disease. We reviewed prosthesis survival, patient survival, complication rates, functional outcomes, and rates of conversion to THA.

Results Five of 86 patients (5.8\%) required revision of the femoral component. Five-, 10-and 20-year implant survivorships were $93 \%, 84 \%$, and $56 \%$, respectively. All patients with low-grade disease survived; the 5-year survival rate for patients with metastatic disease was $16 \%$; the $5-, 10-$, and 20 -year survival for IIA/IIB patients was $54 \%$, $50 \%$, and $44 \%$, respectively. Five of 86 patients $(5.8 \%)$ underwent conversion to THA for groin pain.

Conclusions Cemented bipolar proximal femoral replacements after tumor resection proved a durable reconstruction technique. The implants outlived patients with metastatic disease and high-grade localized disease while patients with low-grade disease outlived their implants. The survival of modular prostheses was comparable to that of older, one-piece custom designs.

Level of Evidence Level IV, therapeutic study. See Guidelines for Authors for a complete description of levels of evidence.

\section{Introduction}

Austin Moore and Harold Bohlman [22] published the first case of endoprosthetic reconstruction for a bone neoplasm of the proximal femur in 1943. Nonetheless, amputation remained the mainstay of surgical treatment for proximal femoral tumors until the advent of doxorubicin and methotrexate in the mid-1970s [16]. Despite the fact that limb salvage is now performed in up to $80 \%$ to $90 \%$ of patients with musculoskeletal tumors, there remains debate as to which reconstruction modality allows the greatest function and is most durable [4, 6]. Allograft-prosthetic composite 
and endoprostheses are the two main reconstruction alternatives, but the low incidence of these tumors coupled with often short-term patient followup has made definitive recommendations elusive $[2,14]$. Critics of both reconstruction modalities cite unacceptably high rates of infection and implant failure [2,4]. As long-term patient survival rates continue to improve, concerns regarding long-term durability of both constructs have surfaced.

It is difficult to draw conclusions from the existing literature on long-term outcomes of bipolar proximal femoral replacements (PFRs) because most of the reports are small, heterogeneous cohorts with limited followup [1, 12, 26]. Bickels et al. [1] reported a $7.7 \%$ revision rate in 39 PFRs at a mean followup of 80 months, but the cohort was small and included both unipolar and bipolar prostheses. Menendez et al. [21] reported a 3.2\% revision rate in 62 bipolar PFRs, but the mean followup was only 18 months. Recently, Finstein et al. [11] reviewed a larger cohort of 62 bipolar PFRs in tumor patients and demonstrated good long-term survivorship of the prosthesis (19.4\% revision rate at a mean of 59.2 months' followup). However, none of these studies has reviewed prosthetic survival stratified by tumor stage, a major factor in providing prognostic information to the individual patient. Additionally, all prior reports describe a surprising $0 \%$ conversion rate from bipolar PFR to THA in tumor patients $[1,12,21,26]$.

We therefore asked the following questions: (1) What is the long-term survival of cemented bipolar PFRs? (2) How does prosthesis survival compare to patient survival among patients with Stage I, II, and III disease, respectively? (3) Do newer, modular implants outperform older, custombuilt prostheses? (4) What are the limb salvage rate, functional outcome, and complication rate for PFRs? (5) Do bipolar prostheses require conversion to THAs?

\section{Patients and Methods}

We retrospectively identified all 106 bipolar PFRs implanted in 98 patients between December 1982 and December 2008. Of the 106 implants, 20 were excluded: nine were revision procedures, seven were performed for reasons other than tumor (ie, total joint revisions and trauma), and four were expandable prostheses used in skeletally immature patients. The expandable prostheses were placed with the intent to perform multiple expansions and/or revision procedures at a later date, and these results have been previously reported [7]. The cohort was therefore formed by the remaining 86 primary cemented bipolar PFRs performed in skeletally mature adults with primary tumors. The mean age was 44.5 years (range, $10-83$ years) at the time of surgery. The diagnoses at the time of the index procedures are listed (Table 1). Of the 86 patients, 46
Table 1. Diagnosis on admission and classification based on grade and stage

\begin{tabular}{lcclc}
\hline Index diagnosis & $\begin{array}{l}\text { High } \\
\text { grade }\end{array}$ & $\begin{array}{l}\text { Low } \\
\text { grade }\end{array}$ & $\begin{array}{l}\text { Stage III } \\
\text { primary or } \\
\text { metastatic }\end{array}$ & Total \\
\hline Chondrosarcoma & 9 & 12 & & 21 \\
Osteosarcoma & 17 & 1 & 1 & 18 \\
Ewing's sarcoma & 10 & 4 & & 11 \\
Giant cell tumor & & & & 4 \\
Malignant fibrous & 3 & & & 3 \\
$\quad$ histiocytoma & & & 2 & 2 \\
Fibrosarcoma & 2 & & 1 & 2 \\
Lymphoma & & 1 & & 1 \\
Multiple myeloma & & 1 & & 1 \\
Chondroblastoma & & & 19 & 3 \\
Hemangioma & & 20 & 23 & 86 \\
Soft tissue sarcoma & 2 & & & \\
Metastasis to bone & & 43 & &
\end{tabular}

$(53.5 \%)$ were female and $40(46.5 \%)$ were male. Seven of the 86 patients were lost to followup at 5, 6, 12, 21, 24, 80, and 86 months. The minimum followup was 3 months (mean, 64.4 months; range, 3-291 months; median, 30.7 months). No patients were recalled specifically for this study; information was obtained via chart review. We had prior approval from our institution's Office for Protection of Research Subjects.

Of the 86 implants, 56 were manufactured by Stryker/Howmedica (Mahwah, NJ), 19 by Techmedica Inc (Camarillo, CA), eight by DePuy Orthopaedics, Inc (Warsaw, IN), two by Intermedics Orthopedics, Inc (Austin, TX), and one by Zimmer, Inc (Warsaw, IN). From 1982 to 1990, 32 implanted prostheses were custom-designed onepiece implants designed by the senior authors (JMK, JJE). The remaining 54 implants since 1990 were manufactured using forged stems and titanium modular segments.

Bipolar PFRs were used as the exclusive reconstruction method after tumor resection from the proximal femur since 1982, as our institution had a poor experience with allograft reconstruction [6]. Contraindications included tumors with extension prohibiting distal femoral fixation or adequate soft tissue coverage for a functional extremity. Since 1992, a displaced pathologic fracture has been considered a relative contraindication.

Preoperative planning consisted of a comprehensive history and physical examination, routine laboratory assessment, high-quality plain radiographs, and a CT and/ or MRI to determine the local extent of the lesion. Neoadjuvant radiation was used in 23 patients: nine for metastatic disease, 10 with localized high-grade Ewing's sarcoma, two with high-grade localized soft tissue 
sarcoma, and two with localized high-grade osteosarcoma who presented with a fracture. Neoadjuvant chemotherapy was used in 42 patients: one with low-grade osteosarcoma with pathologic fracture, 36 with high-grade localized disease, and five with metastatic disease.

All surgery was performed by a single surgeon (JJE). The surgical technique was identical in all cases and followed standard principles of surgical oncology $[5,25]$. An extended lateral incision was used for all surgeries and appropriate tissues were retained over the tumor in an attempt to obtain a wide tumor margin. Tumors with large extraosseous extension required additional soft tissue resection. The lateral incision was extended to the anterior superior iliac spine for tumors with anterior soft tissue extension; for posterior tumors, the incision was extended from the greater trochanter to the posterior iliac crest for tumors. The fascia lata was split in line with its fibers and the vastus lateralis was then released proximally. The dissection was continued anterior and posterior to expose the hip. The interval between the quadriceps and the biceps was identified and perforating vessels were ligated. The bulk of the quadriceps was then reflected anteriorly off the femur, the biceps muscle reflected posteriorly, and the external rotators transected. The abductors were then released and reflected proximally, and the joint capsule incised. The hip was then dislocated. The level of resection was marked on the femur with an osteotome. After the osteotomy was completed, the distal marrow was sampled and a frozen-section analysis performed to confirm the absence of tumor at the level of the osteotomy. The acetabulum was then sized and a bipolar component was selected. The femoral canal was reamed, cleaned, and dried. If the tip of the stem ended within the diaphysis of the femur, a cement restrictor was used. In cases in which the stem ended in the distal $8 \mathrm{~cm}$ of the metaphysis, crossstem pin fixation was used (Fig. 1). A trial reduction with all components was then performed. Before cementation, $100 \mathrm{mg}$ Solu-Cortef ${ }^{\mathbb{R}}$ (Pfizer, New York, NY) was given intravenously to protect against the inflammatory effects of fat emboli that can occur during cementation. Antibioticimpregnated cement was used in all cases. With the prosthesis now assembled, the stem was inserted slowly into the femoral canal with the intent to achieve $20^{\circ}$ of neck anteversion. After hip relocation, leg lengths were once again verified, and the leg was taken through a ROM to confirm stability. Modular neck segments were used to increase or decrease leg length accordingly. Meticulous closures were performed as the retained capsule was secured around the femoral neck with nonabsorbable suture. The quadriceps was then pulled over the prosthesis and the proximal portion was sutured to the external rotators and to the remaining biceps muscle. The abductors were pulled distally out to length and, with the hip

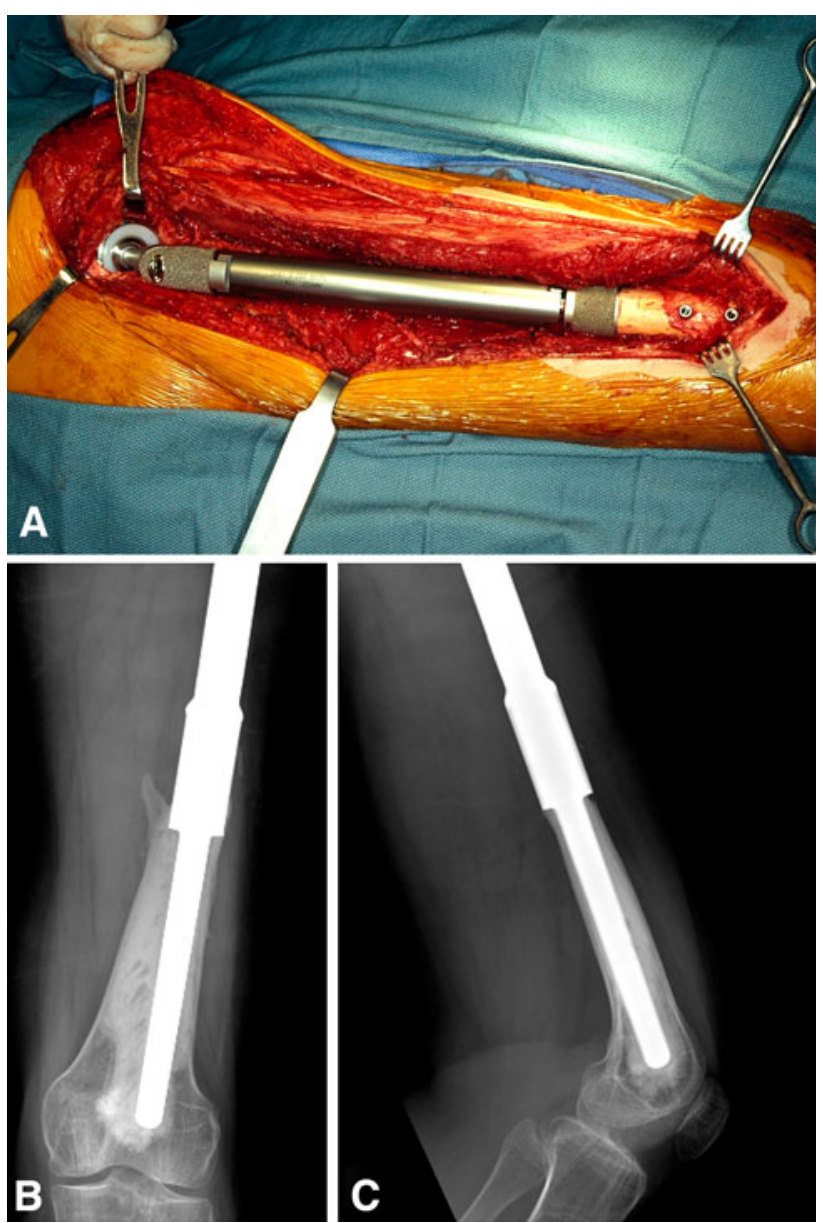

Fig. 1A-C (A) An intraoperative view shows a newer, modular cemented bipolar PFR with circumferential porous coating and distal locking screws as the implant ends in the metaphysis. (B) AP and (C) lateral radiographs of the distal femur show a well-fixed, cemented custom bipolar PFR stem at 26 years after surgery.

abducted, sutured over the vastus lateralis and vastus medialis origins. No vigorous attempts were made to suture the abductors to the prosthesis to simulate anatomic trochanteric attachment. Deep and superficial drains were placed and the wound closed.

Postoperatively, patients were originally placed on bed rest for 4 weeks. Over time, this period of bed rest has been reduced to 7 to 10 days. Antibiotics were continued until the drains were removed. Warfarin was used for anticoagulation postoperatively for 21 days with a target international normalized ratio of 2.0 to 3.0. Several days before discharge, patients began ambulation in an abduction hip brace, which was encouraged for 3 months, at which point weightbearing was permitted as tolerated. Isometric exercises were encouraged initially, along with passive ROM. Abduction strengthening exercises were not begun until 6 weeks and were then carried on for 3 to 6 months. 
Patients were seen in clinic 2, 4, 8, and 12 weeks postoperatively and then every 3 months for the first 2 years. At these visits, all patients underwent wound checks and ROM testing, AP and lateral radiographs of the proximal femur were taken, and in patients with neoplastic disorders, a chest radiograph was taken. Chest CT scans were performed quarterly for patients with high-grade localized disease. From 2 to 4 years from surgery, patients were followed biannually with a clinical examination and hip and chest radiographs. Chest CT scans were obtained biannually in this group. After 4 years, the patients were seen annually with the same physical examination and radiographs in perpetuity. Cross-sectional imaging such as CT and MRI was only used at the operative site if the clinical examination and/or radiographs were suggestive of local relapse.

Functional outcome was assessed using the system adopted by the Musculoskeletal Tumor Society (MSTS) for the functional evaluation of reconstructive procedures after skeletal resection [8]. The MSTS scoring system is a clinician-scored 30-point scale assessing each of six categories (pain, stability, strength, appearance, acceptance, and function) with scores of 0 to 5 . Scores were considered excellent if five of six variables scored 5 points, good if five variables scored 3 points or more, fair if five of six factors scored 1 or more points, and poor if two or more factors scored 0 . Scores were available for 53 of the 86 patients.

Prosthesis survivorship was defined by revision of the cemented component for any reason (including mechanical failure, infection, or local recurrence) as an end point. Mechanical failure included aseptic loosening or fatigue fracture of the femoral component. The rate of successful limb salvage was determined using amputation for any reason as an end point. All other repeat surgical procedures, including conversion to THA, were considered separately, as the cemented stem was retained.

To determine disease-specific patient and prosthetic survival rates, the study cohort of 86 reconstructions was divided into three groups defined by the staging system described by Enneking [8, 9]: Group $1(\mathrm{n}=20)$ included those with low-grade malignancy (Stage IA or IB) or benign conditions; Group $2(n=43)$ consisted of those with high-grade localized disease (Stage IIA or IIB); and Group $3(n=23)$ consisted of those with Stage III primary sarcomas, lymphoma, myeloma, or metastatic disease.

Patient, prosthesis, and limb survival rates were determined using the Kaplan-Meier product-limit method [15]. For the purpose of the Kaplan-Meier analysis, all patients were evaluated based on their status at their most recent clinic visit. Therefore, patients who died of disease with an intact implant were counted as nonrevised implants at the time point of their last clinic visit. Patients who were eventually lost to followup were included in the statistical evaluation based on their status at their last clinic visit. In either case, the analysis included the contribution of these individuals only up to the point of their last known status, ie, length of followup. Survival curves were compared using the log-rank method [19]. Statistical analysis was performed using a commercially available statistics package (SPSS ${ }^{\circledR}$ Version 11.0; SPSS Inc, Chicago, IL).

\section{Results}

Five of the 86 patients $(5.8 \%)$ underwent revision of their prostheses, as defined as removal of the femoral component. Three underwent revision for aseptic loosening, one for deep infection, and one underwent removal of a wellfixed stem because of osteolysis at the prosthesis-stem junction at the time of conversion to THA (Table 2). Overall prosthesis survival rates at 5,10 , and 20 years were thus $97 \%, 84 \%$, and $56 \%$, respectively (Table 3 ).

Patient survival was vastly different among patients with Stage I, II, and III disease. For patients with low-grade tumors $(n=20)$, there was $100 \%$ disease-specific survival at 20 years. The 5-year disease-specific survival rate for Stage III primary sarcoma, metastatic tumors, lymphoma, and multiple myeloma $(\mathrm{n}=23)$ was $15.5 \%$, and no patients in this group survived to 10 years. The 5-, 10-, and 20-year disease-specific survival rates for patients diagnosed with high-grade localized disease $(\mathrm{n}=43)$ were 54\%, 50\%, and 44\%, respectively (Table 3; Fig. 2). Comparing these rates to the prosthetic survival rates, we found the reconstructions outlived patients with metastatic disease (Stage III) and high-grade localized disease (Stage

Table 2. Patients requiring revision of femoral component $(n=5)$

\begin{tabular}{lllllr}
\hline Patient & Age (years) & Index diagnosis & Stage & Cause of revision & Time to revision (months) \\
\hline 1 & 42 & Chondrosarcoma & IA & Aseptic loosening & 63 \\
2 & 19 & Chondroblastoma & IA & Osteolysis of prosthesis-stem junction & 200 \\
3 & 22 & Ewing's sarcoma & IIB & Aseptic loosening & 48 \\
4 & 40 & Osteosarcoma & IIB & Aseptic loosening & 244 \\
5 & 49 & Lymphoma & III & Deep infection & 85 \\
\hline
\end{tabular}


Table 3. Kaplan-Meier 5-, 10-, and 20-year survivorship data for implant, patient, and limb

\begin{tabular}{|c|c|c|c|c|c|c|c|c|c|}
\hline \multirow[t]{3}{*}{ Survivorship } & \multicolumn{3}{|c|}{5 years } & \multicolumn{3}{|c|}{10 years } & \multicolumn{3}{|c|}{20 years } \\
\hline & \multirow[t]{2}{*}{$\%$} & \multicolumn{2}{|c|}{$95 \%$ confidence interval } & \multirow[t]{2}{*}{$\%$} & \multicolumn{2}{|c|}{$95 \%$ confidence interval } & \multirow[t]{2}{*}{$\%$} & \multicolumn{2}{|c|}{$95 \%$ confidence interval } \\
\hline & & Lower & Upper & & Lower & Upper & & Lower & Upper \\
\hline \multicolumn{10}{|l|}{ Implant survival } \\
\hline Low grade $(n=20)$ & 90.9 & 82.2 & 99.6 & 90.9 & 82.2 & 99.6 & 68.2 & 47.5 & 88.9 \\
\hline Stage IIA/IIB $(n=43)$ & 93.8 & 87.6 & 99.9 & 93.8 & 87.8 & 99.8 & 46.9 & 13.6 & 80.2 \\
\hline Stage III $(\mathrm{n}=23)$ & 100 & & & NA & & & NA & & \\
\hline Custom $(\mathrm{n}=32)$ & 94.1 & 88.4 & 99.8 & 87.4 & 79 & 95.8 & 58.3 & 33.9 & 82.7 \\
\hline Modular $(\mathrm{n}=54)$ & 100 & & & 85.7 & 72.5 & 98.9 & NA & & \\
\hline Overall $(n=86)$ & 96.9 & 93.9 & 99.9 & 83.6 & 75.9 & 91.3 & 55.7 & 32.4 & 79 \\
\hline \multicolumn{10}{|c|}{ Patient survival by diagnosis } \\
\hline Low grade $(\mathrm{n}=20)$ & 100 & & & 100 & & & 100 & & \\
\hline Stage IIA/IIB $(n=43)$ & 53.8 & 45.6 & 62 & 50.2 & 41.8 & 58.6 & 44 & 34.6 & 53.6 \\
\hline Stage III $(\mathrm{n}=23)$ & 15.5 & 6 & 25 & NA & & & NA & & \\
\hline Limb salvage $(\mathrm{n}=86)$ & 100 & & & 95.7 & 91.4 & 100 & 95.7 & 91.4 & 100 \\
\hline
\end{tabular}

$\mathrm{NA}=$ not applicable.

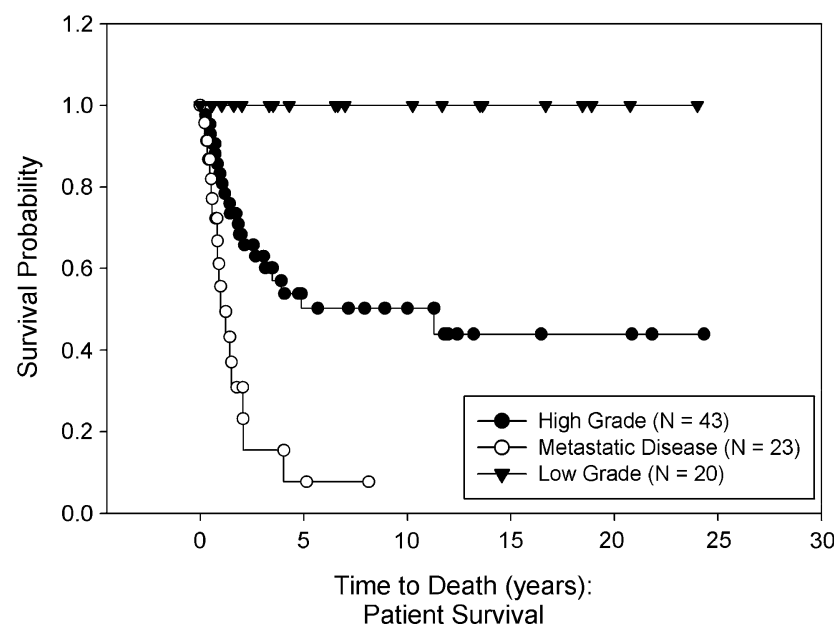

Fig. 2 Kaplan-Meier curves show postoperative patient survival for patients undergoing bipolar PFRs for tumors as divided by tumor stage at the time of diagnosis. This graph illustrates the stark differences in survival based on grade and stage of disease.

IIA/B), while patients with low-grade disease outlived their implants ( $\mathrm{p}<0.05)$ (Table 3; Fig. 3).

We found no difference $(\mathrm{p}=0.582)$ in prosthesis survival between modular and nonmodular implants (Table 3; Fig. 4).

One of the 86 patients (1.2\%) underwent amputation, a hemipelvectomy performed at 87.7 months for a local recurrence. The remaining 85 patients $(98.8 \%)$ maintained a viable limb, producing a $95.7 \%$ limb survival rate at 20 years. Six other patients had local recurrences treated with palliative care. Functional evaluation data were available on 53 of 86 patients at a mean 92 months

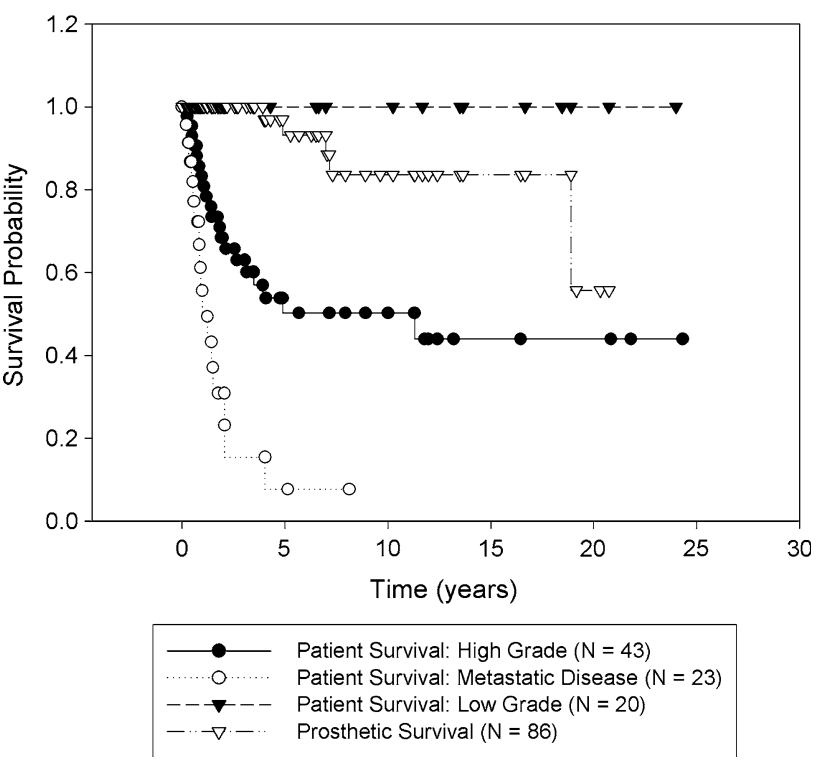

Fig. 3 Kaplan-Meier curves show postoperative patient survival based on tumor stage versus postoperative prosthesis survival. This graph illustrates these prostheses outsurvive patients with metastatic and high-grade disease, whereas patients with low-grade disease outsurvive the prostheses.

postoperatively (range, 7-288 months). The mean MSTS score was $87.0 \%$ (mean raw score, 26.2; range, 13-30), and function was considered excellent in 15 patients $(28.3 \%)$, good in $29(54.7 \%)$, fair in nine $(17.0 \%)$, and poor in none. Four of 86 patients $(4.6 \%)$ experienced systemic complications (nonfatal pulmonary embolism, posttransfusion hepatitis, distant acute myelogenous leukemia, and postoperative perforated diverticulum). Eighteen local complications occurred in 16 patients (18.6\%) (Table 4). 
Dislocation occurred in four patients $(4.6 \%)$. The single cases of wound dehiscence and seroma formation were surgically débrided in the immediate postoperative period and required no further care. The single case of deep infection was in an immunosuppressed lymphoma patient 85 months after her index surgery and was managed with a

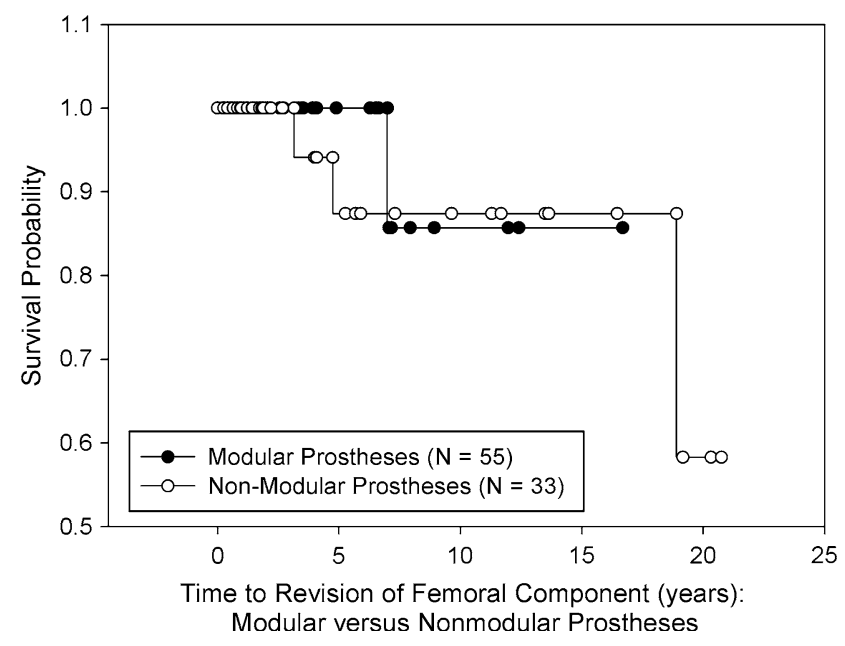

Fig. 4 Kaplan-Meier curves of postoperative prosthesis survival compare older, one-piece prostheses to newer, custom implants. We found no difference in survival between modular and nonmodular prostheses. staged prosthesis exchange. We noted no mechanical fatigue fractures or nerve palsies.

Five of $86(5.8 \%)$ patients underwent conversion to THA for groin pain (Table 5). All patients reported resolution of symptoms with the revision procedure. None of the 23 patients who underwent adjuvant radiation therapy required conversion to THA.

\section{Discussion}

As advances in chemotherapy, radiation therapy, and limb salvage have led to survival improvements in patients with malignancies of the proximal femur, successful surgical intervention has been redefined to include not only preservation of life and limb but also a durable, reliable, and functional extremity. Long-term outcome studies of cemented bipolar PFRs are thus needed to evaluate these prostheses and compare them to other reconstruction modalities available. Specifically, questions of prosthetic survival as compared to patient survival, complications of the surgery, and conversions to THA must be answered to develop realistic expectations for the implants. While studies of long-term outcomes in oncology patients are notoriously difficult due to often shortened life

Table 4. Local complications in patients undergoing primary proximal femoral replacement for oncologic disease

\begin{tabular}{|c|c|c|c|c|}
\hline Local complication & $\begin{array}{l}\text { No further surgery } \\
\text { required }\end{array}$ & $\begin{array}{l}\text { Requiring surgery without revision } \\
\text { of stemmed component }\end{array}$ & $\begin{array}{l}\text { Requiring revision } \\
\text { of a stemmed component }\end{array}$ & Total \\
\hline Local relapse & $5 * *$ & 2 & & 7 \\
\hline Dislocation & & 4 & & 4 \\
\hline $\begin{array}{l}\text { Aseptic loosening } \\
\text { of femoral component }\end{array}$ & & & $4^{*}$ & 4 \\
\hline Deep infection & & & 1 & 1 \\
\hline Wound dehiscence & & 1 & & 1 \\
\hline Seroma & & 1 & & 1 \\
\hline Nerve palsies & & & & 0 \\
\hline Fatigue fractures & & & & 0 \\
\hline Total & & 8 & 5 & 18 \\
\hline
\end{tabular}

* One was well fixed but showed osteolysis at the prosthesis-stem interface.

** Treated with palliative care.

Table 5. Patients requiring conversion to THA $(n=5)$

\begin{tabular}{|c|c|c|c|c|c|}
\hline Patient & Index diagnosis & Age (years) & Radiation? & Chemotherapy? & Time to conversion (months) \\
\hline 1 & Giant cell tumor & 16 & No & No & 49 \\
\hline 2 & Giant cell tumor & 44 & No & No & 13 \\
\hline 3 & Osteosarcoma & 15 & No & Yes & 71 \\
\hline 4 & Chondrosarcoma & 43 & No & No & 33 \\
\hline 5 & Osteosarcoma & 19 & No & Yes & 244 \\
\hline
\end{tabular}


expectancies, this study of 86 patients has a mean of followup of more than 65 months.

Our study is associated with several limitations. First, we lacked control patients managed with allograft-composite implants for comparison. As our early institutional allograft experience was poor [6], endoprosthetic reconstruction has been used exclusively at our institution since 1982. Despite this, our study provides outcomes to which institutions that perform allograft-composite can compare their results. Second, we compared an aggregate prosthesis survival curve to patient survival curves for patients with Stage I, II, and III disease. We believe the similarity in prosthesis survival among the different tumor stage groups allows this (Table 3) and the aggregate prosthesis survival curve provides the most robust curve for comparison.

Our overall revision rate of $5.8 \%$ compares to that of other reports of PFRs. Bickels et al. [1] reported a revision rate of $7.7 \%$ at a mean of 80 months while Farid et al. [10] reported a revision rate of $13.5 \%$ with a median followup of 146 months. Menendez et al. [21] reported a 3.2\% revision rate, but followup was only 18.1 months. In their review of bipolar PFRs, Finstein et al. [11] reported a revision rate of $19.4 \%$ (Table 6).

By stratifying patients according to disease-specific life expectancy, our results help to answer a key question regarding prosthesis longevity: is the patient likely to outlive his or her prosthesis? For patients with Stage IIA or IIB disease or patients with Stage III disease, the survival of the implants exceeded patient survival. Patients with low-grade or benign disease, however, outlived their prostheses. Therefore, these patients should be counseled preoperatively that, while bipolar PFRs provide a durable reconstruction, they likely would require a revision surgery at some point in their lifetime.

We found no difference in survival between newer, modular prostheses and older, one-piece custom prostheses. While Finstein et al. [11] found modular PFRs outperformed custom designs, our 10-year survival rates were nearly identical (87.4\% and $85.7 \%$ ). While our sample size of 32 custom prostheses was larger than the 10 described by Finstein et al. [11], additional studies will be required to provide a definitive answer to this question.

Our high limb preservation rate $(98.8 \%)$ and good functional results (83\% good or excellent MSTS scores) are consistent with the literature. Malawar et al. [20] and Finstein et al. [11] both also reported one single case of amputation in their respective cohorts. Bickels et al. [1] reported $87 \%$ good or excellent results in 39 patients, while Finstein et al. [11] reported an MSTS mean of 21.3 with $71 \%$ good or excellent results in 13 patients. While the subjectivity of the MSTS system and the variation of reporting methods make comparison across studies difficult [18], these results indicate reliably good to excellent function can be obtained with PFRs. It should be noted, however, we had six additional cases of local relapse before 1992 treated palliatively, of which five presented with pathologic fractures. This led us to conclude local control cannot be consistently achieved with this procedure in patients presenting with a displaced pathologic fracture due to tumor seeding of the surrounding compartment; therefore, since 1992, these patients were recommended amputation for local control.

Five of 86 patients (5.8\%) required conversion to THA for groin pain and/or acetabular cartilage loss. While not reported in the oncology literature, the limitations of bipolar hemiarthroplasties have been well documented in other patient populations [23, 24]. In patients with degenerative arthritis, Pellegrini et al. [24] reported a $12 \%$ conversion to THA in long-term followup. In patients with hemiarthroplasty for fracture, Ong et al. [23] reported an $18 \%$ rate of moderate to severe groin pain. In osteonecrosis patients with bipolar hemiarthroplasty, incidences of groin pain have been reported from $12 \%$ to $42 \%$ [3, 13, 17], which is perhaps the best comparison group as they resemble tumor patients in that they are generally younger and have no pathology of the acetabulum. Ito et al. [13]

Table 6. A comparison to prior publications reporting on PFRs in oncologic patients

\begin{tabular}{|c|c|c|c|c|c|c|}
\hline Study & $\begin{array}{l}\text { Number of } \\
\text { implants }\end{array}$ & $\begin{array}{l}\text { Mean followup } \\
\text { (months) }\end{array}$ & Type of reconstruction & $\begin{array}{l}\text { Conversion to } \\
\text { THA }\end{array}$ & Revisions & Limb salvage rate \\
\hline Bickels et al. [1] & 39 & 80 & Bipolar and unipolar & 0 & $7.7 \%(3 / 39)$ & $98 \%(45 / 46)$ \\
\hline Farid et al. [10] & 52 & 146 (median) & $\begin{array}{l}40 \text { bipolar, } 12 \text { acetabular } \\
\text { resurface }\end{array}$ & 0 & $13.5 \%(7 / 52)$ & \\
\hline Finstein et al. [11] & 62 & 59.2 & Bipolar PFR & 0 & $19.4 \%(12 / 62)$ & $98.4 \%(61 / 62)$ \\
\hline Horowitz et al. [12] & 16 & 80 & $\begin{array}{l}\text { Bipolar } \pm \text { acetabular } \\
\text { cement }\end{array}$ & & & \\
\hline Menendez et al. [21] & 62 & 18.1 & Bipolar PFR & 0 & $3.2 \%(2 / 62)$ & \\
\hline Zeegen et al. [27] & 57 & 18 & Unknown & & $0 \%$ & \\
\hline Bernthal et al. & 86 & 65.3 & Bipolar PFR & $5.8 \%(5 / 86)$ & $7.0 \%(6 / 86)$ & $98.9 \%(85 / 86)$ \\
\hline
\end{tabular}

PFR = proximal femoral replacement. 
reported $6.3 \%$ of bipolar hemiarthroplasties for osteonecrosis required conversion to THA. The five patients in our study who required conversion to THA were younger than our average patient (mean 27.5 versus 44.5 years) and perhaps placed more physical demands on the reconstruction. Three of the patients did not receive adjuvant chemotherapy and all five did not receive radiation, suggesting these are not predisposing factors for conversion to THA in our experience.

This study represents the largest reported series of cemented bipolar PFRs implanted for tumors. By stratifying patients by tumor grade and stage, the data demonstrate patients with metastatic and high-grade tumors are unlikely to require revision surgery, whereas patients with lowgrade tumors will likely outlive their prosthesis. The 5.8\% conversion rate to THA, which is unique to this series, suggests groin pain and acetabular erosion be carefully evaluated in patients who have undergone bipolar PFRs. Still, with a $5.8 \%$ overall revision rate and a $99 \%$ limb salvage rate, we conclude cemented bipolar PFRs provide a durable method of reconstruction for the vast majority of patients with musculoskeletal tumors.

Acknowledgments We thank Frederick R. Eilber, MD, and Fritz C. Eilber, MD, for their contribution in the care of these patients, the development of our tumor database, and their review of this manuscript.

Open Access This article is distributed under the terms of the Creative Commons Attribution Noncommercial License which permits any noncommercial use, distribution, and reproduction in any medium, provided the original author(s) and source are credited.

\section{References}

1. Bickels J, Meller I, Henshaw RM, Malawar MM. Reconstruction of hip stability after proximal and total femur resections. Clin Orthop Relat Res. 2000;375:218-230.

2. Brien EW, Terek RM, Healy JH, Lane JM. Allograft reconstruction after proximal tibial resection for bone tumors: an analysis of function and outcome comparing allograft and prosthetic reconstructions. Clin Orthop Relat Res. 1994;303:116-127.

3. Cabanela ME. Bipolar versus total hip arthroplasty for avascular necrosis of the femoral head: a comparison. Clin Orthop Relat Res. 1990;261:59-62.

4. Cannon CP, Zeegan E, Eckardt JJ. Techniques in endoprosthetic reconstruction. Oper Tech Orthop. 2005;14:225-235.

5. Eckardt JJ, Eilber FR. Endoprosthetic replacement. Curr Orthop. 1993;7:148-156.

6. Eckardt JJ, Eilber FR, Grant TO, Mirra JM, Weisenberger TH, Dorey FJ. Management of Stage IIB osteogenic sarcoma: experience at the University of California, Los Angeles. In: Cancer Treatment Symposia. Vol 3. National Institutes of Health Publication Number 85-2766. Bethesda, MD: National Institutes of Health; 1985:117-130.

7. Eckardt JJ, Kabo JM, Kelley CM, Ward WG Sr, Asavamongkolkul A, Wirganowicz PZ, Yang RS, Eilber FR.
Expandable endoprosthesis reconstruction in skeletally immature patients with tumors. Clin Orthop Relat Res. 2000;373:51-61.

8. Enneking WF. Modification of the system for functional evaluation of surgical management of musculoskeletal tumors. In: Limb Salvage in Musculoskeletal Oncology. Enneking WF, ed. New York, NY: Churchill Livingstone; 1987:626-639.

9. Enneking WF, Spanier SS, Goodman MA. A system for the surgical staging of musculoskeletal sarcoma. Clin Orthop Relat Res. 1980;153:106-120.

10. Farid Y, Lin PP, Lewis VO, Yasko AW. Endoprosthetic and allograft-prosthetic composite reconstruction of the proximal femur for bone neoplasms. Clin Orthop Relat Res. 2006;442:223229.

11. Finstein J, King J, Fox E, Ogilvie C, Lackman R. Bipolar proximal femoral replacement prostheses for musculoskeletal neoplasms. Clin Orthop Relat Res. 2007;459:66-75.

12. Horowitz SM, Glasser DB, Lane JM, Healey JH. Prosthetic and extremity survivorship after limb salvage for sarcoma: how long do the reconstructions last? Clin Orthop Relat Res. 1993;293: 280-286.

13. Ito $\mathrm{H}$, Matsuno $\mathrm{T}$, Kaneda $\mathrm{K}$. Bipolar hemiarthroplasty for osteonecrosis of the femoral head. Clin Orthop Relat Res. 2000;374:201-211.

14. Jeys LM, Kulkarni A, Grimer RJ, Carter SR, Tillman RM, Abudu A. Endoprosthetic reconstruction for the treatment of musculoskeletal tumors of the appendicular skeleton and pelvis. $J$ Bone Joint Surg Am. 2008;90:1265-1271.

15. Kaplan EL, Meier P. Nonparametric estimation from incomplete observation. J Am Stat Assoc. 1958;53:457-481.

16. Lange B, Kramer S, Gregg J, Toledano S, Wimmer R, Evans A. High-dose methotrexate and Adriamycin in osteogenic sarcoma: The Children's Hospital of Philadelphia Study. Am J Clin Oncol 1982;5:3-8.

17. Lee S, Sugano N, Nakata K, Matsui M, Ohzono K. Comparison between bipolar hemiarthroplasty and THA for osteonecrosis of the femoral head. Clin Orthop Relat Res. 2004;424:161-165.

18. Lieberman JR, Dorey F, Shakelle P, Schumaker L, Thomas BJ, Kilgus DJ, Finerman GA. Differences between patients' and physicians' evaluations of outcome after total hip arthroplasty. J Bone Joint Surg Am. 1996;78:835-888.

19. Machin D, Palmar MKB. Comparison of 2 survival curves. In Machin D, Palmar MKB, eds. Survival Analysis: A Practical Approach. Chichester, England: Bookcraft; 1995:66-75.

20. Malawar MM, Chou LB. Prosthetic survival and clinical results with use of large-segment replacements in the treatment of highgrade bone sarcomas. J Bone Joint Surg Am. 1995;77:1154-1165.

21. Menendez LR, Ahlmann ER, Kermani C, Gotha H. Endoprosthetic reconstructions for neoplasms of the proximal femur. Clin Orthop Relat Res. 2006;450:46-51.

22. Moore AT, Bohlman HR. Metal hip joint: a case report. $J$ Bone Joint Surg Am. 1943;25:688-692.

23. Ong B, Maurer S, Aharonoff G, Zuckerman J, Koval K. Unipolar versus bipolar hemiarthroplasty: functional outcome after femoral neck fracture at a minimum of thirty-six months of follow up. J Orthop Trauma. 2002;16:317-322.

24. Pellegrini VD Jr, Heiges BA, Bixler B, Lehman EB, Davis CM III. Minimum ten-year results of primary bipolar hip arthroplasty for degenerative arthritis of the hip. J Bone Joint Surg Am. 2006;88:1817-1825.

25. Springfield DS, Enneking WF, Neff JR, Makley JT. Principles of tumor management. Instr Course Lect. 1984;33:1-25.

26. Zeegen EN, Aponte-Tinao LA, Hornicek FJ, Gebhardt MC, Mankin HJ. Survivorship analysis of 141 modular metallic endoprostheses at early followup. Clin Orthop Relat Res. 2004; 420:239-250. 